\title{
Gal's Accurate Tables Method Revisited
}

\author{
Damien Stehlé, Paul Zimmermann
}

$\mathbf{N}^{\circ} 5359$

Octobre 2004

THÈME 2 



\title{
Gal's Accurate Tables Method Revisited
}

\author{
Damien Stehlé, Paul Zimmermann \\ Thème 2 - Génie logiciel \\ et calcul symbolique \\ Projet Spaces \\ Rapport de recherche $n^{\circ} 5359$ - Octobre $2004-23$ pages
}

\begin{abstract}
Gal's accurate tables algorithm aims at providing an efficient implementation of elementary functions with correct rounding as often as possible. This method requires an expensive pre-computation of a table made of the values taken by the function - or by several related functions - at some distinguished points. Our improvements of Gal's method are two-fold: on the one hand we describe what is the arguably best set of distinguished values and how it improves the efficiency and correctness of the implementation of the function, and on the other hand we give an algorithm which drastically decreases the cost of the pre-computation. These improvements are related to the worst cases for the correct rounding of mathematical functions and to the algorithms for finding them. We show that the whole method can be turned into practice by giving complete tables for $2^{x}$ and $\sin x$ for $x \in\left[\frac{1}{2}, 1[\right.$, in double precision.
\end{abstract}

Key-words: Gal's method, elementary functions, lattice reduction, simultaneous worst cases. 


\section{Améliorations de la méthode de Gal}

Résumé : L'algorithme de Gal a pour but de fournir une implantation efficace des fonctions mathématiques élémentaires avec arrondi correct aussi souvent que possible. Cette méthode nécessite un pré-calcul très coûteux d'une table de valeurs prises par la fonction considérée - ou de plusieurs fonctions en relation avec celle-ci en un ensemble de points distingués. Nous améliorons la méthode de Gal de deux manières: d'une part nous décrivons quel est le meilleur ensemble de points distingués et comment il améliore l'efficacité et la correction de l'implantation de la fonction, et d'autre part nous donnons un algorithme qui décroit considérablement le coût du pré-calcul. Ces améliorations sont liées aux pires cas pour l'arrondi correct des fonctions mathématiques et aux algorithmes qui les calcule. Nous montrons que nos améliorations sont raisonnables en pratique en donnant des tables complètes pour $2^{x}$ et $\sin x$ avec $x \in\left[\frac{1}{2}, 1\right.$ [, en double précision.

Mots-clés : Méthode de Gal, fonctions mathématiques élémentaires, réduction de réseaux, pires cas simultanés. 


\section{Introduction}

The IEEE-754 standard for floating-point arithmetic [12] specifies that the four basic arithmetic operations and the square root should be correctly rounded, but does not require a correct rounding for any other elementary mathematical function, like trigonometric, exponential and logarithmic functions. For this reason, there are libraries which produce incorrectly rounded results, or which do not guarantee a correctly rounded result, and, for example, this causes serious difficulties for the portability and reproducibility of scientific calculations. The reticence to extend the standard to the elementary mathematical functions comes from the fact that an implementation guaranteeing a correct rounding is too expensive. Citing David Hough in the context of the revision of the IEEE-754 standard $^{1}$ :

[Elementary functions] are too hard to standardize now because nobody knows how to trade-off precision vs performance. If less than correct rounding is specified [...] then [it] is always possible that somebody will come up with a non-standard algorithm that is more accurate AND faster. This can't happen with a correct rounding specification, but correctly-rounded transcendental functions seem to be inherently much more expensive than almost-correctly-rounded. One could instead standardize properties that approximate functions are supposed to obey - but anomalies still abound. All these points argue against standardizing transcendental functions now.

In the present paper, we improve a routine commonly used in the implementation of elementary functions, namely Gal's accurate tables method (see [8,9] and [17] pp58-62), in the hope it will shrink the efficiency gap between elementary functions libraries in use and those guaranteeing a correct rounding (such as [1,25]), and give more support for proposals of standardization of these functions $[18,7]$.

The implementation of an elementary function over its full domain for some given precision usually requires two phases (see [6] for the exponential function): a quick phase giving a correctly rounded result for an overwhelming proportion of the entries and an accurate phase which is considerably slower but performed only in the few cases for which the quick phase was not sufficient. The quick phase often uses the input-output precision as working precision (or eventually extends the precision or uses a few more bits in very few steps), while the accurate phase is often based on Ziv's strategy [24] which extends the working precision until the result can be guaranteed correctly rounded (eventually, one may also use a sharp bound on the

\footnotetext{
${ }^{1}$ http://grouper. ieee.org/groups/754/meeting-minutes/01-03-14.html
} 
required precision if such a bound is known $[13,14])$. The quick phase is itself often subdivided into three sub-phases:

- First range reduction: The full domain of the function is restricted to a smaller one by using the mathematical properties of the considered function, e.g. $\exp (x+$ $k \ln 2)=2^{k} \cdot \exp (x)$ giving the range $\left[0, \ln 2\left[, 2^{x+k}=2^{k} \cdot 2^{x}\right.\right.$ giving the range $[0,1[$, $\sin \left(x+k \frac{\pi}{2}\right)=f_{k}(x)$ with $f_{k}= \pm \sin x$ or $f_{k}= \pm \cos x$ depending on $k \bmod 4$, giving the range $\left[-\frac{\pi}{4}, \frac{\pi}{4}[, \ldots\right.$

- Second range reduction: By a table lookup, the range is shrunk further. For example, we write $2^{x}=2^{x_{0}} \cdot 2^{h}$, (resp. $\sin x=\sin x_{0} \cdot \cos h+\cos x_{0} \cdot \sin h$ ) where $\left(x_{0}, 2^{x_{0}}\right)$ (resp. $\left(x_{0}, \sin x_{0}, \cos x_{0}\right)$ ) is stored in the table and $h=x-x_{0}$ is small $(|h|$ is approximately smaller than the length of the range obtained after the first reduction, divided by the number of distinguished elements). We call related functions the functions used in this range reduction: for $2^{x}$ there is one related function (namely $2^{x}$ ), but for $\sin x$ there are two related functions (namely $\sin x$ and $\cos x$ ).

- Polynomial evaluation: The remaining terms $\left(\right.$ e.g. $\left.2^{h}, \cos h, \sin h, \ldots\right)$ are evaluated by using a polynomial approximation of the function (or the related functions) over the restricted range.

There are very satisfactory answers for the first [20] and last sub-phases [2]. Gal's method addresses the second sub-phase. The original technique is based on a table of "almost regularly spaced" distinguished points which images by the function (or the related functions) are unusually close to machine numbers. The table, of size a few kilobytes [5], is obtained via a pre-computation based on a naive search.

We improve Gal's method in two ways. First we notice that the best set of distinguished points is made of the values for which the function (resp. the related functions) is hard to round (resp. to round simultaneously) in the case of directed rounding (towards 0 or $\infty$ ): this problem is therefore closely related to the Table Maker's Dilemma (TMD for short). A direct consequence of this fact is that we can adapt the methods which find the worst cases of a one-variable function [13, $14,22,23]$ in order to construct the tables, if there is a single related function. This is much more efficient than Gal's naive search. In the case of one related function, Gal's method can be adapted for the accurate phase to guarantee a correct rounding for any input. The second improvement is an algorithm to construct the table when there are two related functions: we modify the lattice-based algorithm of $[22,23]$ to find simultaneously bad cases for two functions.

In the paper, although the method can be easily generalized to other functions and to arbitrary precision, we focus on $2^{x}$ and $\sin x$ for $x \in\left[\frac{1}{2}, 1\right.$ [ in double precision.

INRIA 
For this choice of parameters, our improvements towards Gal's original method are the following:

1. The table for $\sin x$ is constructed much more efficiently than by naive search (the cost of the naive search would have been $2^{52}$ calls to $\sin x$ and $\cos x$ in extended precision).

2. For $\sin x$, the proportion of entries for which the quick phase fails decreases from $\approx 2^{-10}$ to $\approx 2^{-20}$.

3. The accurate phase for $2^{x}$ can be based on Gal's method by using the work of Lefèvre $[13,14]$. It requires only quadruple precision calculations.

The rest of the paper is organized as follows. In Section 2 we recall some basic facts about the TMD and Gal's accurate tables method. In Section 3 we describe what is the best set of distinguished values in the table and how this choice improves the quick and accurate phases. In Section 4 we explain how to obtain these tables. Finally, in Section 5 we show that the method is practical for double precision by giving parts of the tables for $2^{x}$ and $\sin x$.

Notations: We define $[|a, b|]$ (resp. $[\mid a, b[\mid$ ) as the set of integers in $[a, b]$ (resp. $[a, b[$ ). For any integer $n,\left[a, b\left[{ }_{n}\right.\right.$ denotes the set of the $\frac{m}{2^{n}}$ 's where $a \leq \frac{m}{2^{n}}<b$ and $m$ is an integer. For example, $\left[\frac{1}{2}, 1[53\right.$ corresponds to the doubles with exponent -1 . For any reals $x$ and $c, x \operatorname{cmod} c$ denotes $x-\left\lfloor\frac{x}{c}\right\rceil$, and if $\frac{x}{c}$ is half an odd integer, we choose any of the possibilities. In particular, $x$ cmod 1 is the "centered" fractional part of $x$. We denote by $o(x)$ a machine number closest to $x$ in double precision. Finally, vectors are denoted in bold and for a vector $\mathbf{x} \in \mathbb{R}^{n},\|\mathbf{x}\|$ and $\|\mathbf{x}\|_{1}$ are respectively its $L_{2}$ and $L_{1}$ norms, i.e. $\left\|\left(x_{1}, \ldots, x_{n}\right)\right\|=\sqrt{\sum_{i=1}^{n} x_{i}^{2}}$ and $\left\|\left(x_{1}, \ldots, x_{n}\right)\right\|_{1}=\sum_{i=1}^{n}\left|x_{i}\right|$.

\section{Preliminaries}

This section gives the necessary background to describe our improvements of Gal's accurate tables method. We describe an informal model where the functions we consider are regarded as black-boxes returning uniformly distributed outputs. Experiments corroborate very well this model. We use it to describe Gal's original scheme and to give the basic ideas concerning the Table Maker's Dilemma.

\subsection{The Random Model}

We consider that a function behaves as a random black-box when the first bits of its output are thrown away. Such an assumption is of course very strong and completely

$\mathrm{RR} \mathrm{n}^{\circ} 5359$ 
heuristic. Nevertheless experiments tend to validate this hypothesis and in particular the experiments of Section 5 do not contradict it.

A given function $f:\left[\frac{1}{2}, 1\left[\rightarrow\left[a, b\left[\right.\right.\right.\right.$ for some $a<b\left(\right.$ e.g. $2^{x}:\left[\frac{1}{2}, 1[\rightarrow[1,2[)\right.$ is seen as a random black-box: for any $n$ and $k_{1}<k_{2}$ larger than some thresholds, if $x$ is chosen randomly and uniformly in $\left[\frac{1}{2}, 1\left[_{n}\right.\right.$, then the bits at positions $k_{1}$ and $k_{2}$ of the binary expansion of $f(x)$ are independent random variables uniformly distributed in $\{0,1\}$. This implies some useful properties:

- The probability of obtaining a run of $p$ consecutive identical bits- starting from some given position - in the binary expansion of $f(x)$ is $2^{1-p}$.

- If we consider $2^{p}$ consecutive $x$ 's, there is $O(1)$ of them such that the binary expansion of $f(x)$ has a run of $p$ consecutive identical bits starting from some given position.

- The set of the $x \in\left[\frac{1}{2}, 1[n\right.$ such that $f(x)$ has a run of $p$ consecutive identical bits - starting from some given position - is of cardinality around $2^{n-p}$, and the maximum distance between two consecutive elements is less than $\approx\left(1+n-\frac{p}{2}\right)$. $2^{n-p}$ (see the appendix for a proof).

We generalize this model to two functions $f_{1}, f_{2}:\left[\frac{1}{2}, 1[\rightarrow[a, b[\right.$ for some $a<b$ $(e . g . \sin x$ and $\cos x)$. They are seen as random independent black-boxes: firstly they both are black-boxes, and secondly, for any $n$ and $k_{1}, k_{2}$ larger than some thresholds, if $x$ is chosen randomly and uniformly in $\left[\frac{1}{2}, 1\left[n\right.\right.$, then the bit at position $k_{1}$ of the binary expansion of $f_{1}(x)$ and the bit at position $k_{2}$ of the binary expansion of $f_{2}(x)$ are independent random variables. From such an hypothesis we can also derive some properties:

- The probability of obtaining a run of $p$ consecutive identical bits- starting from some given position - in the binary expansions of both $f_{1}(x)$ and $f_{2}(x)$ is $2^{2-2 p}$.

- If we consider $2^{2 p}$ consecutive $x$ 's, there is $O(1)$ of them such that the binary expansions of both $f_{1}(x)$ and $f_{2}(x)$ have a run of $p$ consecutive identical bits starting from some given position.

- The set of the $x \in\left[\frac{1}{2}, 1{ }_{n}\right.$ such that $f_{1}(x)$ and $f_{2}(x)$ have a run of $p$ consecutive identical bits starting from some given position is of cardinality around $2^{n-2 p}$, and the maximum distance between two consecutive elements is less than $\approx$ $(1+n-p) \cdot 2^{n-2 p}$.

Notice that it is easy to make all these statements rigorous and to generalize the model to more than two functions.

INRIA 


\subsection{Gal's Accurate Tables Method}

Gal's accurate tables method [8,9] addresses the second range reduction of the quick phase of the calculation of $f(x)$. The method is general, but here we take as examples $f(x)=2^{x}$ and $f(x)=\sin x$. The idea of the second range reduction is to write:

$$
\begin{gathered}
2^{x}=2^{x_{0}} \cdot 2^{h}, \\
\sin x=\sin x_{0} \cdot \cos h+\cos x_{0} \cdot \sin h,
\end{gathered}
$$

where $x_{0}$ is in a table of distinguished points and is stored with an approximation of its corresponding $2^{x_{0}}$ (resp. $\sin x_{0}$ and $\cos x_{0}$ ); $h=x-x_{0}$ is small: roughly speaking, $|h|$ is smaller than $\frac{1}{2}$ divided by the number of distinguished points. After this tablebased range reduction, $2^{h}$ (resp. $\cos h$ and $\sin h$ ) is computed approximately by using a low degree polynomial (resp. two low degree polynomials) which closely approximates $2^{h}$ (resp. $\sin h$ and $\cos h$ ) when $h$ is close to 0 . To fix the ideas, we can suppose that these polynomials are the Taylor expansions of the functions at 0 , but it is possible to do better [2]. If we consider the double precision (i.e. 53 bits of mantissa), since we are in the quick phase, the calculations should be made in double precision as often as possible, and it is interesting to have tables with approximately $2^{10}$ distinguished elements, for cache optimization reasons (see [5, 1]).

The naive way of choosing the distinguished elements is to take them equally spaced, thus minimizing the maximum value taken by $|h|$. Gal's idea is to relax very slightly the maximum value taken by $|h|$ in order to choose better distinguished points: he takes almost regularly spaced distinguished points $x_{0}$ such that the stored approximation of $2^{x_{0}}$ (resp. $\sin x_{0}$ and $\cos x_{0}$ ) is unusually close to its true value. More precisely, in double precision, for any $0 \leq i<2^{10}, x_{0}^{i}$ is a machine number closest to $\frac{1}{2}+\frac{i}{2^{11}}$ such that:

$$
\begin{gathered}
\left|2^{52} \cdot 2^{x_{0}^{i}} \operatorname{cmod} 1\right| \leq 2^{-10} \text { for } 2^{x} \\
\left|2^{53+e} \cdot \sin x_{0}^{i} \operatorname{cmod} 1\right| \leq 2^{-10} \text { and }\left|2^{53} \cdot \cos x_{0}^{i} \operatorname{cmod} 1\right| \leq 2^{-10} \text { for } \sin x,
\end{gathered}
$$

where $e=1$ if $\left|x_{0}^{i}\right| \leq \frac{\pi}{6}$ and 0 otherwise. According to the random model, $x_{0}^{i}$ should be extremely close to $\frac{1}{2}+\frac{i}{2^{11}}$, implying a negligible increase of the value that can be taken by $|h|$. Moreover, this set of distinguished points gives a more accurate implementation of the function: since $\left|x-x_{0}\right|$ is bounded by $\approx 2^{-12}$, the final output for $f(x)$ can be made accurate with $\approx 63$ bits of precision although the calculations are made with doubles. Again in the random model, this implies that the value 
output for $f(x)$ is correctly rounded with probability around $1-2^{-10}$, which is higher than $99.9 \%$.

The tables are constructed by exhaustive search, so that there are approximately $2^{9}$ trials for any distinguished element of the $2^{x}$-table, and approximately $2^{18}$ trials for any distinguished element of the $\sin x$-table.

\subsection{Some Reminders on the TMD}

Let $n$ be the precision and $f:\left[\frac{1}{2}, 1\left[\rightarrow\left[\frac{1}{2}, 1[\right.\right.\right.$ be the considered function. A $m$-bad case $x$ for $f$ is a $n$-bit machine number such that at least $n+m$ bits are needed to round $f(x)$ correctly. More precisely these are the $x$ 's in $\left[\frac{1}{2}, 1[n\right.$ such that:

$-\left|2^{n} \cdot f(x) \operatorname{cmod} 1\right| \leq 2^{-m}$ for the directed rounding modes (towards $0,+\infty$ or $-\infty)$,

- $\mid 2^{n} \cdot f(x)-\frac{1}{2}$ cmod $1 \mid \leq 2^{-m}$ for the rounding to nearest mode.

Notice that in this definition, $m$ can be any real number. Here the functions we consider have output ranges different from $\left[\frac{1}{2}, 1[\right.$, so we adopt the definition to our case. We say that $x \in\left[\frac{1}{2}, 1\left[53\right.\right.$ is a $m$-bad case for $2^{x}$ if $\left|2^{52+x} \operatorname{cmod} 1\right| \leq 2^{-m}$, a $m$-bad case for $\cos x$ if $\left|2^{53} \cdot \cos x \operatorname{cmod} 1\right| \leq 2^{-m}$, a $m$-bad case for $\sin x$ if $\mid 2^{53}$. $\sin x \operatorname{cmod} 1 \mid \leq 2^{-m}$ when $\left.x \in\right] \frac{\pi}{6}, 1\left[\right.$ and $\left|2^{54} \cdot \sin x \operatorname{cmod} 1\right| \leq 2^{-m}$ if $x \in\left[\frac{1}{2}, \frac{\pi}{6}[\right.$.

The knowledge of the hardness to round $f$, i.e. the maximum $m$ such that $f$ admits a $m$-bad case, makes it possible to implement $f$ efficiently, because the maximum number of bits which are needed is known in advance. The drawback of this approach is that this value is hard to compute. The exhaustive search is by far infeasible because of its $\approx 2^{n}$ complexity. Lefèvre and Muller proposed $\mathrm{a} \approx 2^{2 n / 3}$ algorithm [13, 14], which was sufficient to perform some systematic work in double precision. Stehlé, Lefèvre and Zimmermann [22] gave a generalization of this algorithm by using lattice-based Coppersmith's work to find small roots of modular polynomials [3,4]. They obtained an algorithm with a heuristic $\approx 2^{3 n / 5}$ complexity, which was later improved to $\approx 2^{5 n / 7}$ [23]. Although these complexity bounds are better than the one of Lefèvre's algorithm, it seems that the practical cut-off between both methods is around the double extended precision, i.e. $n=64$.

\section{$3 \quad$ Gal's Accurate Tables Method Revisited}

Our improvement is based on the idea that it is possible to require runs of more than 10 consecutive identical bits as Gal does. We first describe what are the best sets of distinguished points for $2^{x}$ and $\sin x$, and then show that for $2^{x}$, Gal's method based

INRIA 
on this set can be used for the whole evaluation scheme (both quick and accurate phases).

\subsection{A Table Made of Bad Cases for the Directed Rounding Modes}

Under the random model assumption, it is possible to strengthen the requirements of Gal on the lengths of the runs of consecutive identical bits. For the $2^{x}$ function, we can take as set of distinguished points all the 42-bad cases. There are approximately $2^{12}$ of them and the maximum distance between two consecutive ones is below $2^{-9.914}$, see Section 5. Choosing such a set of distinguished values decreases the error made on $2^{x_{0}}$ in

$$
2^{x}=2^{x_{0}} \cdot 2^{h}
$$

but since the maximum distance between two distinguished values is not decreased, the error made while evaluating the polynomial approximating $2^{h}$ for $h$ close to 0 is roughly the same, i.e. $\approx 2^{-10}$ : since the coefficients of the polynomial are doubles and since this is also the working precision, the degree- 1 coefficient is correct with error bounded by $\approx 2^{-53-10}$. As a consequence, the error made in the quick phase is roughly the same. But as we will see in the next subsection, this choice of distinguished points makes the accurate phase more efficient.

For the $\sin x$ function, since there are two related functions in the second range reduction (namely $\sin x$ and $\cos x$ ), we cannot expect runs of consecutive identical bits as long as for $2^{x}$. Indeed, the best choice of distinguished values is made of all the $x$ 's that are simultaneously 21-bad cases for $\sin x$ and $\cos x$. There are approximately $2^{12}$ of them, and the average distance between two consecutive ones is below $2^{-9.977}$ as shown by the experiments of Section 5 . This means that the errors made on $\sin x_{0}$ and $\cos x_{0}$ in

$$
\sin x=\sin x_{0} \cdot \cos h+\cos x_{0} \cdot \sin h
$$

are decreased from $\approx 2^{-63}$ to $\approx 2^{-74}$. We argue that the errors made in the polynomial evaluations corresponding to $\cos h$ and $\sin h$ can also be made that small, in a very efficient way. We define

$$
S(h)=h-a_{3} h^{3}+a_{5} h^{5} \text { and } C(h)=1-\frac{1}{2} h^{2}+a_{4} h^{4},
$$

where $a_{i}$ is the double closest to $\frac{1}{i !}$ for $i \in\{3,4,5\}$. Using the fact that $|h| \leq 2^{-10.977}$ along with Lagrange's bound, we obtain that:

$$
|\sin h-S(h)| \leq\left|\sin h-\left(h-\frac{h^{3}}{6}+\frac{h^{5}}{120}\right)\right|+\left|a_{3}-\frac{1}{6}\right||h|^{3}+\left|a_{5}-\frac{1}{120}\right|\left|h^{5}\right|
$$

$\mathrm{RR} \mathrm{n}^{\circ} 5359$ 


$$
\begin{aligned}
& \leq 2^{-76.839} \cdot 2^{-12.299}+2^{-56.584} \cdot 2^{-32.931}+2^{-62.906} \cdot 2^{-54.885} \\
& \leq 2^{-88.314} \\
|\cos h-C(h)| & \leq\left|\cos h-\left(1-\frac{h^{2}}{2}+\frac{h^{4}}{24}\right)\right|+\left|a_{4}-\frac{1}{24}\right||h|^{4} \\
& \leq 2^{-65.862} \cdot 2^{-9.491}+2^{-58.584} \cdot 2^{-43.908} \\
& \leq 2^{-75.352} .
\end{aligned}
$$

We now explain how to evaluate equation (1). Let $c_{0}$ and $s_{0}$ be the two machine numbers approximating $\cos x_{0}$ and $\sin x_{0}$. By construction we have $\left|c_{0}-\cos x_{0}\right|, \mid s_{0}-$ $\sin x_{0} \mid \leq 2^{-74}$. Let $C^{*}(h)=C(h)-1$ and $S^{*}(h)=S(h)-h$. Recall that $h \leq 2^{-10.977}$. We first compute approximations of $S^{*}(h)$ and $C^{*}(h)$ with Horner's method in double precision:

$$
\begin{array}{rrr}
k \leftarrow o\left(h^{2}\right) & \left|k-h^{2}\right| \leq 2^{-54-21} \leq 2^{-75} & k \leq 2^{-21.953} \\
k^{\prime} \leftarrow o(h \cdot k) & \left|k^{\prime}-h^{3}\right| \leq 2^{-10.977-75}+2^{-54-32} \leq 2^{-84.988} & \left|k^{\prime}\right| \leq 2^{-32.930} \\
S^{*} \leftarrow o\left(a_{5} \cdot k\right) & \left|S^{*}-a_{5} \cdot h^{2}\right| \leq 2^{-6.906-75}+2^{-54-28} \leq 2^{-80.952} & \left|S^{*}\right| \leq 2^{-28.859} \\
S^{*} \leftarrow o\left(S^{*}-a_{3}\right) & \left|S^{*}-\left(-a_{3}+a_{5} \cdot h^{2}\right)\right| \leq 2^{-80.952}+2^{-54-2} \leq 2^{-55.999} & \left|S^{*}\right| \leq 2^{-2.584} \\
S^{*} \leftarrow o\left(k^{\prime} \cdot S^{*}\right) & \left|S^{*}-S^{*}(h)\right| \leq 2^{-84.988-2.584}+2^{-55.999-32.930}+2^{-54-35} \leq 2^{-86.754} & \left|S^{*}\right| \leq 2^{-35.513} \\
C^{*} \leftarrow o\left(a_{4} \cdot k\right) & \left|C^{*}-a_{4} \cdot h^{2}\right| \leq 2^{-4.584-75}+2^{-54-26} \leq 2^{-78.777} & \left|C^{*}\right| \leq 2^{-26.536} \\
C^{*} \leftarrow o\left(C^{*}-\frac{1}{2}\right) & \left|C^{*}-\left(-\frac{1}{2}+a_{4} \cdot h^{2}\right)\right| \leq 2^{-78.777}+2^{-54-2} \leq 2^{-55.999} & \left|C^{*}\right| \leq 2^{-1.000} \\
C^{*} \leftarrow o\left(k \cdot C^{*}\right) & \left|C^{*}-C^{*}(h)\right| \leq 2^{-75-1.000}+2^{-55.999-21.953}+2^{-54-22} \leq 2^{-74.824} & \left|C^{*}\right| \leq 2^{-22.951} .
\end{array}
$$

The calculation of $\sin x$ ends with the sum:

$$
s=s_{0}+c_{0} \cdot h+\left(s_{0} \cdot C^{*}+c_{0} \cdot S^{*}\right),
$$

the term " $c_{0} \cdot h$ " being evaluated in an extended precision, for example by using a quadruple, a double-double, or by simulating the extended precision with the use of a fma operation. For example we do this with a fma.

$-h^{\prime} \leftarrow o_{f m a}\left(s_{0}+c_{0} \cdot h\right)$.

$-t \leftarrow o\left(h^{\prime}-s_{0}\right)$.

$-l \leftarrow o_{f m a}\left(t-c_{0} \cdot h\right)$.

Since $\left|c_{0} \cdot h\right| \leq \frac{s_{0}}{2}$, we have $h^{\prime} \geq \frac{s_{0}}{2}$ and the second operation is exact. Therefore we have:

$h^{\prime}+l=s_{0}+c_{0} \cdot h$ and $\left|\left(h^{\prime}+l\right)-\left(\sin x_{0}+\cos x_{0} \cdot h\right)\right| \leq 2^{-74}+2^{-74-10.977} \leq 2^{-73.999}$.

We now compute some less significant bits, which in particular suffice to round correctly the result most of the time.

\begin{tabular}{c|ccc}
$S^{*} \leftarrow o\left(c_{0} \cdot S^{*}\right)$ & $\left|S^{*}-\cos x_{0} \cdot S^{*}(h)\right| \leq 2^{-74-35.513}+2^{-0.188-86.754}+2^{-54-35} \leq 2^{-86.631}$ & $\left|S^{*}\right| \leq 2^{-35.700}$ \\
$C^{*} \leftarrow o\left(s_{0} \cdot C^{*}\right)$ & $\left|C^{*}-\sin x_{0} \cdot C^{*}(h)\right| \leq 2^{-74-22.951}+2^{-0.249-74.667}+2^{-54-23} \leq 2^{-74.610}$ & $\left|C^{*}\right| \leq 2^{-23.199}$
\end{tabular}

INRIA 


$$
\begin{array}{cc}
C^{*} \leftarrow o\left(S^{*}+C^{*}\right) & \left|C^{*}-\left(\cos x_{0} \cdot S^{*}(h)+\sin x_{0} \cdot C^{*}(h)\right)\right| \leq 2^{-86.631}+2^{-74.610} \leq 2^{-74.609} \\
C^{*} \leftarrow o\left(l+C^{*}\right) & \left|\left(h^{\prime}+C^{*}\right)-\sin x\right| \leq 2^{-74.609}+2^{-73.999}+2^{-1-106} \leq 2^{-73.271} .
\end{array}
$$

When the distance between $2^{53+e} \cdot \sin x$ and $\mathbb{Z}$ is higher than $2^{53+e} \cdot 2^{-73.271}=$ $2^{-19.271+e}$ - where $e$ is 1 in the case of a rounding to the nearest mode, and 0 for a directed rounding mode - the addition $h^{\prime}+C^{*}$ gives the correct output. This implies that by using such a scheme the result is correctly rounded with probability at least $1-2^{-19.271}$, which makes the $99.9 \%$ estimate of Gal increase to $\approx 99.9998 \%$.

\subsection{A Function Evaluation Scheme Based on Gal's Method}

In this subsection we describe how one could evaluate $2^{x}:\left[\frac{1}{2}, 1[\rightarrow[1,2[\right.$ in double precision with correct rounding by using only Gal's method, in the case of a directed rounding mode. As usual, there are two phases: the quick phase and the accurate phase.

The quick phase We use Gal's method with the table made of the 42-bad cases. This induces an error bounded by $2^{-41}$ for the term $2^{x_{0}}$, and the problem is reduced to computing approximately $2^{h}$ where $|h|$ is smaller than $2^{-10.914}$ (see Section 5). We do this by evaluating the polynomial $P(h)=1+a_{1} h+\ldots+a_{4} h^{4}$, where $a_{i}$ is the double that is closest to $\frac{(\ln 2)^{i}}{i !}$. It is possible to check that $P(h)$ can be evaluated with only operations on doubles to approximate $2^{h}$ with error bounded by $\approx 2^{-63}$ when $|h| \leq 2^{-10.914}$. This implies that if $x$ is not a 10-bad case, the value calculated so far for $2^{x}$ is correct. Moreover, as a side effect, if $x$ is a 42 -bad case, the result can also be correctly rounded: for each entry $\left(x_{0}, 2^{x_{0}}\right)$ of the table, we add a bit of information telling whether the stored value for $2^{x_{0}}$ is slightly larger or smaller than its real value.

The accurate phase Suppose now that the quick phase was not sufficient to guarantee a correct rounding of the output. We keep the same pair $\left(x_{0}, 2^{x_{0}}\right)$ and only change the polynomial evaluation sub-phase. We evaluate in quadruple precision the polynomial $Q(h)=1+b_{1} h+\ldots+b_{7} h^{7}$ where $b_{i}$ is the quadruple precision machine number that is closest to $\frac{(\ln 2)^{i}}{i !}$. It can be checked that $Q(h)$ can be evaluated with operations on quadruples to approximate $2^{h}$ with error bounded by $2^{-106.821}$ when $|h| \leq 2^{-10.914}$. Let $\bar{Q}(h)$ be the value computed for $Q(h)$, and $y_{0}$ be the value for $2^{x_{0}}$ that is stored in the table. We finally approximate $2^{x}$ by $y_{0} \cdot \bar{Q}(h)$, the error being bounded by:

$$
2^{-106.821+1}+2^{-41-53} \cdot 1.000360+2^{-114+1} \leq 2^{-93.999} .
$$

$\mathrm{RR} \mathrm{n}^{\circ} 5359$ 
This implies that if $x$ is not a 41.999-bad case, then the result is rounded correctly. Therefore, we have an implementation returning the correct rounding for any input $x$ except those that are 41.999-bad cases but not 42-bad cases. We fix this point in the following way. We consider a table made only of 42.00072-bad cases instead of 42-bad cases, which means that we remove $6543825232174115 / 2^{53}$ from the look-up table and consider it as a special case. This removal does not change the bound on $|h|$. Moreover, it is easy to see that the error analysis just above now gives an final error bounded by $2^{-94}$. Therefore the result is correctly rounded because we must be in one of the three following situations: $x$ is not a 42-bad case, the error bound gives that the value returned for $2^{x}$ is correctly rounded; $x$ is a 42.000072 -bad case, the output is stored in the table; otherwise $x$ is $6543825232174115 / 2^{53}$.

\section{The Computation of the Tables}

So far, we built our function evaluation scheme as if we knew explicitly the tables we described. Nevertheless, the task of computing the tables is by far nontrivial: if we were using an exhaustive search as Gal does, the cost of computing the table of either $2^{x}$ or $\sin x$ would be $2^{52}$ calls to $2^{x}$ (resp. $\sin x$ ) in extended precision. For $2^{x}$ the problem is easily solved once it is noted that the 42-bad cases can be calculated by Lefèvre's algorithm $[13,14]$ or the lattice-based algorithm of Stehlé, Lefèvre and Zimmermann $[22,23]$. In this section, after giving some background on lattices and lattice reduction algorithms, we describe a method to construct simultaneously bad cases for two functions. For a given precision $n$, this algorithm admits a heuristic

complexity of $\approx 2^{n / 2}$ - instead of $\approx 2^{n}$ for the exhaustive search, and $\approx 2^{2 n / 3}$ with Lefèvre's algorithm.

\subsection{A few Reminders on Lattices}

In this subsection we briefly give some necessary background on lattices and on lattice reduction algorithms. We refer to $[11,16]$ for more details on both the algorithmic and mathematical aspects.

A lattice $L$ is a discrete subgroup of $\mathbb{R}^{n}$, or equivalently the set of all linear integral combinations of $\ell \leq n$ linearly independent vectors $\mathbf{b}_{i}$ over $\mathbb{R}$, that is:

$$
L=\left\{\sum_{i=1}^{\ell} x_{i} \mathbf{b}_{i} \mid x_{i} \in \mathbb{Z}\right\} .
$$

Notice that the rank $\ell$ of the lattice can be smaller than the dimension $n$ of the embedding space. As soon as $\ell \geq 2$, a given lattice $L$ admits an infinity of bases 
related to each other by unimodular transformations. We define the determinant of the lattice $L$ as $\operatorname{det}(L)=\prod_{i=1}^{\ell}\left\|\mathbf{b}_{i}^{*}\right\|$, where $\mathbf{b}_{1}^{*}, \ldots, \mathbf{b}_{\ell}^{*}$ is the Gram-Schmidt orthogonalization of a lattice basis $\mathbf{b}_{1}, \ldots, \mathbf{b}_{\ell}$. This quantity does not depend of the choice of the basis. Most of the time, only bases which consist of short vectors are of interest. The $i$-th minimum of the lattice $L$ is the smallest $r$ such that the ball centered in $\mathbf{0}$ and of radius $r$ contains at least $i$ linearly independent lattice vectors. For example the first minimum $\lambda_{1}(L)$ is the length of a shortest non-zero lattice vector.

Since it corresponds to our situation in the following subsection we now suppose that $\ell=4$ and $L \subset \mathbb{Z}^{5}$. It is classical [11] that in this case there always exists a basis reaching the four first minima, such a basis being called Minkowski-reduced, and that the first minimum of $L$ is below $\sqrt{2} \cdot \operatorname{det}(L)^{1 / 4}$. Moreover, the well-known LLL algorithm [15] can be used to find a vector which is not much longer than the first minimum.

Theorem 1. Given a basis $\left[\boldsymbol{b}_{1}, \ldots, \boldsymbol{b}_{4}\right]$ of a lattice $L \subset \mathbb{Z}^{5}$, if $M=$ max $_{i}\left\|\boldsymbol{b}_{i}\right\|$, the LLL algorithm provides in time $O\left(\log ^{3} M\right)$ a basis $\left[\boldsymbol{v}_{1}, \ldots, \boldsymbol{v}_{4}\right]$ satisfying:

$$
\left\|\boldsymbol{v}_{1}\right\| \leq 4 \lambda_{1}(L) \leq 4 \sqrt{2} \operatorname{det}(L)^{1 / 4} .
$$

This is not optimal because the output basis is not necessary Minkowski-reduced, and therefore the vectors may not be as short as possible. The greedy algorithm of [19] outputs a basis reaching the first four minima, and has the additional advantage of admitting a $O\left(\log ^{2} M\right)$ complexity bound.

\subsection{An Algorithm for the $\sin x$-Table}

The search over $\left[\frac{1}{2}, 1\left[\right.\right.$ is divided into $\frac{N}{2 T}$ quick searches over intervals of length $\frac{T}{N}$. These quick searches are performed by using the algorithm we describe below.

Given two functions $f_{1}$ and $f_{2}$, a precision $n$, a bad-case bound $m$ and a search bound $T$, the following algorithm tries to find all the machine numbers $x \in\left[-\frac{T}{2^{n}}, \frac{T}{2^{n}}\right]$ such that:

$$
\left|2^{n} \cdot f_{i}(x) \operatorname{cmod} 1\right| \leq 2^{-m} \text { for } i \in\{1,2\} .
$$

In fact it solves this problem for any $N$ and $M$ in place of $2^{n}$ and $2^{m}$. It starts by approximating both $f_{1}$ and $f_{2}$ by polynomials, and then tries to solve the problem for these polynomials instead of the initial functions, by using Coppersmith's method to find small roots of multivariate modular polynomials [3,4]. Our algorithm is heuristic: all its outputs are correct, but it may eventually fail. Nevertheless, the heuristic is 
quite reasonable and the algorithm worked very well in our experiments described in Section 5: we follow the strategy of halving $T$ until the algorithm does not fail, and it happens that the average $T$ corresponds to what the theory predicts.

In the algorithm we use a routine LatticeReduce, which can either be the LLL algorithm [15] or the greedy algorithm of [19]. The input functions are made independent of $N: F_{i}(t)=N f_{i}(t / N)$.

Algorithm SimultaneousBadCaseSearch.

Input: Two functions $F_{1}$ and $F_{2}$, and two positive integers $M, T$.

Output: All $t \in[-T, T]$ such that $\left|F_{i}(t) \operatorname{cmod} 1\right|<\frac{1}{M}$ for $i \in\{1,2\}$.

1. Let $P_{1}(t), P_{2}(t)$ be the degree-2 Taylor expansions of $F_{1}(t), F_{2}(t)$.

2. Compute $\varepsilon$ such that $\left|P_{i}(t)-F_{i}(t)\right|<\varepsilon$ for $|t| \leq T$ and $i \in\{1,2\}$.

3. Let $M^{\prime}=\left\lfloor\frac{1 / 2}{1 / M+\varepsilon}\right\rfloor, C=3 M^{\prime}$, and ${ }^{2} \tilde{P}_{i}(\tau)=\left\lfloor C \cdot P_{i}(T \tau)\right\rceil$ for $i \in\{1,2\}$.

4. Let $e_{1}=1, e_{2}=\tau, e_{3}=\tau^{2}, e_{4}=v, e_{5}=\phi$.

5. Let $g_{1}=C, g_{2}=C \cdot \tau, g_{3}=\tilde{P}_{1}(\tau)+3 v, g_{4}=\tilde{P}_{2}(\tau)+3 \phi$.

6. Create the $4 \times 5$ integral matrix $L$ where $L_{k, l}$ is the coefficient of the monomial $e_{l}$ in $g_{k}$.

7. $V \leftarrow$ LatticeReduce $(L)$

8. Let $\mathbf{v}_{1}, \mathbf{v}_{2}, \mathbf{v}_{3}$ be the three shortest vectors of $V$, and $Q_{i}(\tau)$ the associated polynomials.

9. If there exists $i \in\{1,2,3\}$ such that $\left\|\mathbf{v}_{i}\right\|_{1} \geq C$, return(FAIL).

10. Let $Q(\tau)$ be a linear combination of the $Q_{i}$ 's which is independent of $v$ and $\phi$. We have $\operatorname{deg} Q \leq 1$.

11. Let $q(t)=Q\left(\frac{t}{T}\right)$. For each $t_{0}$ in $\operatorname{IntegerRoots}(q,[-T, T])$ do if $\left|F_{i}\left(t_{0}\right) \operatorname{cmod} 1\right|<\frac{1}{M}$ for all $i \in\{1,2\}$, then output $t_{0}$.

\subsection{Correctness of the Algorithm}

The following theorem gives the correctness of the algorithm.

Theorem 2. In case the algorithm does not return FAIL, it behaves correctly, i.e. it outputs exactly all integers $t \in[-T, T]$ such that $\mid F_{i}(t)$ cmod $1 \mid<\frac{1}{M}$ for $i \in\{1,2\}$.

Proof: Because of the final check in Step 11, we only have to check that no worst case is missed. Suppose there is $t_{0} \in[-T, T]$ with $\left|F_{i}\left(t_{0}\right) \operatorname{cmod} 1\right|<\frac{1}{M}$ for $i \in\{1,2\}$. From the definition of $P_{i},\left|P_{i}\left(t_{0}\right) \operatorname{cmod} 1\right|<\frac{1}{M}+\varepsilon \leq \frac{1}{2 M^{\prime}}$. Since $\left|C \cdot P_{i}(T \tau)-\tilde{P}_{i}(\tau)\right| \leq \frac{3}{2}$ for $|\tau| \leq 1$, by choosing $\tau_{0}=\frac{t_{0}}{T}$ we get $\left|\tilde{P}_{i}\left(\tau_{0}\right) \operatorname{cmod} C\right|<3$.

Whence the $g_{i}$ 's have a common root $\left(t_{0} / T, v_{0}, \phi_{0}\right) \in[-1,1]^{3}$, modulo $C$. Since the $Q_{i}$ 's are linear integer combinations of the $g_{i}$ 's, they admit a common root in $[-1,1]^{3}$ modulo $C$, and even over the reals since $\left\|\mathbf{v}_{1}\right\|_{1},\left\|\mathbf{v}_{2}\right\|_{1},\left\|\mathbf{v}_{3}\right\|_{1} \leq C$. Finally $t_{0}$ is an integer root of $q(t)$ and will be found at Step 11.

${ }^{2}$ The notation $\left\lfloor C \cdot P_{i}(T \tau)\right\rceil$ means that we round to the nearest each coefficient of $C \cdot P_{i}(T \tau)$. This gives an element of $\mathbb{Z}[\tau]$.

INRIA 
Working Precision In Step 1, we can use floating-point coefficients in the Taylor expansion $P_{i}(t)$ instead of symbolic coefficients, as long as it introduces no error in Step 3 while computing $\tilde{P}_{i}(\tau)$, for $i \in\{1,2\}$. Let $a_{j}^{i}$ be the $j$-th Taylor coefficient of $f_{i}$. In order to get a correct $\tilde{P}_{i}(\tau)$ at Step 3 , the error on $C N\left(\frac{T}{N}\right)^{j} \cdot a_{j}^{i}$ must be less than $\frac{1}{2}$, thus the error on $a_{j}^{i}$ must be less than $\frac{1}{2 C \cdot N}\left(\frac{N}{T}\right)^{j}$. Since $N \geq T$, it thus suffices to compute $a_{j}^{i}$ with $\log _{2}(2 C N) \leq 2 n$ bits after the binary point. (We will see below that $C=O\left(N^{1 / 2}\right)$.)

\subsection{Complexity Analysis of the Algorithm}

Now that the correctness of the algorithm is proved, we estimate its complexity. Let $a_{0}^{i}, a_{1}^{i}, \ldots$ be the Taylor coefficients of $f_{i}$ for $i \in\{1,2\}$. Here we assume that for any $k$ and $i,\left|a_{k}^{i}\right|=O(1)$.

Taylor's Bound Since we neglect Taylor coefficients of degree three and higher, the error made in the approximation to $N \cdot f_{i}\left(\frac{t}{N}\right)$ by $P_{i}(t)$ is $\approx a_{3}^{i} \cdot T^{3} \cdot N^{-2}$. Since we are looking for simultaneously bad cases with $\left|P_{i}(t) \operatorname{cmod} 1\right|<\frac{1}{M}$, we want $T^{3} \cdot N^{-2}=O\left(\frac{1}{M}\right)$, i.e. $T^{3}=O\left(\frac{N^{2}}{M}\right)$.

The Size of the Coefficients of the $\tilde{\boldsymbol{P}}_{\boldsymbol{i}}$ 's The degree- 2 polynomials $\tilde{P}_{i}(\tau)=\tilde{p}_{0}^{i}+$ $\tilde{p}_{1}^{i} \tau+\tilde{p}_{2}^{i} \tau^{2} \in \mathbb{Z}[\tau]$ computed at Step 3 of the algorithm satisfy $\tilde{p}_{2}^{i}=O\left(M \cdot T^{2} \cdot N^{-1}\right)$. Indeed, since $P_{i}(t)=p_{0}^{i}+p_{1}^{i} t+p_{2}^{i} t^{2}$ is the degree-2 Taylor expansion of $N \cdot f_{i}\left(\frac{t}{N}\right)$, we have $p_{2}^{i}=O\left(N^{-1}\right)$. Moreover, $\tilde{P}_{i}$ is defined by $\tilde{P}_{i}(\tau)=\tilde{p}_{0}^{i}+\tilde{p}_{1}^{i} \tau+\tilde{p}_{2}^{i} \tau^{2}=\left\lfloor C \cdot P_{i}(T \tau)\right\rceil$. The fact that $C=\Theta(M)$ concludes the proof.

The Matrix Computed at Step 6 We have to reduce the lattice spanned by the rows of the following matrix.

$$
L=\left[\begin{array}{lllll}
C & & & \\
& T \cdot C & & \\
\tilde{p}_{0}^{1} & \tilde{p}_{1}^{1} & \tilde{p}_{2}^{1} & 3 \\
\tilde{p}_{0}^{2} & \tilde{p}_{1}^{2} & \tilde{p}_{2}^{2} & 3
\end{array}\right] .
$$

It is easy to compute the determinant of this lattice:

$$
|\operatorname{det}(L)|=3 C^{2} \cdot T \cdot \sqrt{\left(\tilde{p}_{2}^{1}\right)^{2}+\left(\tilde{p}_{2}^{2}\right)^{2}+3}=O\left(M^{3} \cdot T^{3} \cdot N^{-1}\right) .
$$


Coppersmith's Bound In order to ensure the algorithm does not return FAIL at Step 9, we use Theorem 1 to provide at least one short vector: $\operatorname{det}(L)^{1 / 4}$ has to be smaller than $C$, which gives the bound $T^{3}=O(M \cdot N)$. This is not enough to ensure that there are two other short vectors, but it seems that in practice the first three minima for these lattices are most often very similar.

Complexity Analysis We have two bounds for $T, N$ and $M$ :

$$
\begin{array}{ccc}
M \cdot T^{3} & =O\left(N^{2}\right) \quad \text { [Taylor's bound] } \\
T^{3} & =O(M \cdot N) \text { [Coppersmith's bound] }
\end{array}
$$

Since the complexity of the overall search — an exponent range of $N / 2$ values - is approximately poly $(\log N) \cdot \frac{N}{T}$, the best choice of parameters is $T \approx M \approx N^{1 / 2}$, thus giving a heuristic complexity of $\approx \operatorname{poly}(\log N) \cdot N^{1 / 2}$.

Remark: The technique used in Algorithm SimultaneousBadCaseSearch resembles very closely the algorithm of [22,23], but things happen to be somehow simpler. For example, increasing the degree of the polynomial approximations or the dimension of the lattice by taking powers of the $\tilde{P}_{i}$ 's is useless here: it seems that the degree- 2 approximations and the four-dimensional lattice give a better complexity bound than any other choice of parameters.

\section{$5 \quad$ Experimental Results}

We give here the first values of the tables for $2^{x}$ and $\sin (x)$, the complete tables being available at the url: http://www.loria.fr/ stehle/IMPROVEDGAL.html.

\subsection{The Table for $2^{x}$}

We give in Figure 1 the smallest 340 values $t_{0} \in\left[\mid 2^{52}, 2^{53}[\mid\right.$ satisfying:

$$
\left|2^{52+\frac{t_{0}}{2^{53}}} \operatorname{cmod} 1\right| \leq 2^{-m}
$$

with $m \geq 41$. The table has been provided by Vincent Lefèvre and was computed with Lefèvre's algorithm for finding the worst cases of a one-variable function.

There are 4001 elements in the table, 1985 if we choose $m \geq 42,973$ with $m \geq 43$, 491 with $m \geq 44$ and 265 if we choose $m \geq 45$. The maximum distance between two elements of the table is below $2^{-9.914}$, and the worst case is $t_{0}=13 \mathrm{e} 34 \mathrm{fa} 6 \mathrm{ab} 969 \mathrm{e}$ with $m=52$. 


\begin{tabular}{|c|c|c|c|}
\hline$m$ & $t_{0}$ & $t_{0}$ & $t_{0}$ \\
\hline 1 & $3 \mathrm{df} 89 c 941$ & $10 \mathrm{a} 8 \mathrm{c}$ & $10 f 53$ \\
\hline 246 & & $5 \mathrm{~d} 245$ & \\
\hline 942 & b 41 & 46 & 1 \\
\hline 090d6fac990e 43 & $105292757 \mathrm{ba} 15 \mathrm{~b} 41$ & $10 a b 55 c 3 b$ & $10 f t$ \\
\hline 243 & 341 & 449 & 342 \\
\hline Obab73fdcc3f 42 & $10570 d 95 d a$ & 10af78aa5 & $10 f 7218 c$ \\
\hline 0041 & $8 f 43$ & 241 & 1045 \\
\hline 4644 & 5142 & 741 & $10 f$ \\
\hline$f 828 d 746046$ & $1060 \mathrm{e} 7 \mathrm{def}$ & $10 \mathrm{~b} 1880 \mathrm{e}$ & $10 f$ \\
\hline$c 8142$ & $2 \mathrm{e} 42$ & 44 & $3 a 4$ \\
\hline $0 e 0 a 8 c 90 f 68943$ & 106487249 & $10 \mathrm{~b} 290 \mathrm{db}$ & $10 f$ \\
\hline 442 & 842 & 41 & $10 f$ \\
\hline e 142 & 341 & & 642 \\
\hline d12 41 & 1068 & 32dd & $10 f$ \\
\hline dc1 42 & 146 & & \\
\hline efc 41 & $f 41$ & 43 & $10 f$ \\
\hline $50 f 47$ & 31 & & $10 f$ \\
\hline 741 & 41 & & \\
\hline 643 & ef 42 & 44 & $10 f$ \\
\hline & & & \\
\hline 41 & 141 & 42 & 101 \\
\hline & & & \\
\hline 41 & 42 & & 42 \\
\hline 42 & 41 & 42 & 11 \\
\hline 41 & & & 47 \\
\hline a 42 & c 41 & 41 & 11 \\
\hline & & & 742 \\
\hline 941 & 645 & 41 & c 43 \\
\hline 41 & 41 & 41 & c 42 \\
\hline 41 & 141 & 41 & 41 \\
\hline & 243 & & 742 \\
\hline 41 & 843 & & 11 \\
\hline & & 43 & 11 \\
\hline 41 & 644 & 43 & 11 \\
\hline 41 & 3 & 42 & 41 \\
\hline 41 & 42 & & 11 \\
\hline & 41 & & \\
\hline & & & \\
\hline 42 & c 43 & $10 c$ & 110 \\
\hline & 45 & 41 & 11 \\
\hline 46 & e 44 & 741 & $2 \mathrm{~d} 42$ \\
\hline 41 & c 42 & 42 & 941 \\
\hline & & 45 & 43 \\
\hline 43 & 41 & 741 & b 41 \\
\hline & 5642 & c 42 & \\
\hline 1342 & 3845 & $10 \operatorname{cecc} 7 \mathrm{c} 0$ & $11167 \mathrm{c} 6 \mathrm{~b} 40 \mathrm{c} 8 \mathrm{e} 344$ \\
\hline & & & \\
\hline 41 & 1084 & $10 \mathrm{~d} 0489 \mathrm{e}$ & 111 \\
\hline $10310 \mathrm{a} 0 \mathrm{c} 5 \mathrm{a} 76 \mathrm{bd} 41$ & $108608 d 20 c 912 d 42$ & $10 \mathrm{~d} 16 \mathrm{~b} 2 \mathrm{e} 672 \mathrm{e} 9$ & $1119 a 87 f 9 e f 18 a 4$ \\
\hline
\end{tabular}

$\mathrm{RR} \mathrm{n}^{\circ} 5359$ 


\begin{tabular}{|c|c|c|c|}
\hline$m$ & $t_{0}$ & $t_{0}$ & $t_{0}$ \\
\hline 142 & 1087d1ed5a7 & $10 \mathrm{~d} 2$ & 111 \\
\hline 2 ea 41 & 10881b25a3e2d0 41 & $10 \mathrm{~d} 286 \mathrm{a} 02 \mathrm{~d}$ & 1. \\
\hline 843 & 241 & 041 & 42 \\
\hline $33 \mathrm{fb} 60 \mathrm{a}$ & 10896be0d6df11 41 & 10d39996808b21 42 & 111 \\
\hline a 41 & $f 641$ & 7841 & 342 \\
\hline 541 & 0042 & 7741 & \\
\hline $34 d 2 e c c$ & $108 \mathrm{~b} 2 \mathrm{cec}$ & $10 \mathrm{~d} 4 \mathrm{~b} 6 \mathrm{~d} 9 \mathrm{f}$ & 112 \\
\hline d 41 & 108 & 141 & b 42 \\
\hline $37481 c$ & c9a 41 & 10d6ccaed7 & 112 \\
\hline 43 & $28 b 43$ & $10 d$ & 112 \\
\hline 41 & af 41 & 241 & 112 \\
\hline b 41 & lc1 41 & $10 \mathrm{dcOdac}$ & 112 \\
\hline 41 & 108 & & 112 \\
\hline c 41 & c4 41 & $10 \mathrm{~d}$ & 112 \\
\hline & 41 & & \\
\hline 42 & 541 & 41 & 112 \\
\hline a 43 & 741 & $10 e$ & 112 \\
\hline 42 & 43 & & \\
\hline 46 & 042 & $10 \epsilon$ & 11 \\
\hline & & & 112 \\
\hline 41 & 41 & 44 & 112 \\
\hline 41 & 41 & 54 & 11 \\
\hline 42 & 42 & 1 & \\
\hline 42 & 42 & 10 & 11 \\
\hline 43 & 10 & & \\
\hline & 645 & $10 e$ & 11 \\
\hline 41 & 41 & 10 & 113 \\
\hline 42 & $10 s$ & $10 €$ & 11 \\
\hline 842 & 43 & 741 & 113 \\
\hline & 109 & $10 \epsilon$ & \\
\hline & $10 a$ & $10 e$ & 113 \\
\hline 44 & 42 & 10 & 11 \\
\hline 44 & $10 a$ & $10 e$ & 113 \\
\hline f 41 & 79442 & 10 ee017b71576 c 47 & 113 \\
\hline & $10 a$ & & \\
\hline $104 \mathrm{t}$ & 142 & Of514cd3348ee 42 & $1141675 \mathrm{df} 1591 \mathrm{c} 4$ \\
\hline
\end{tabular}

Fig. 1. The smallest 340 entries of the table for $2^{x}$ for double precision and $x \in\left[\frac{1}{2}, 1[\right.$ : for any entry, $t_{0}, m$ satisfy equation (2). 


\subsection{The Table for $\sin x$}

We give in Figure 2 the smallest 340 values $t_{0} \in\left[\mid 2^{52}, 2^{53}[\mid\right.$ satisfying:

$$
\left|2^{53+e} \cdot \sin \frac{t_{0}}{2^{53}} \operatorname{cmod} 1\right| \leq 2^{-m_{1}} \text { and }\left|2^{53} \cdot \cos \frac{t_{0}}{2^{53}} \operatorname{cmod} 1\right| \leq 2^{-m_{2}}
$$

with $m_{1}, m_{2} \geq 21, e=1$ if $\frac{t_{0}}{2^{53}} \leq \frac{\pi}{6}$ and $e=0$ otherwise. The table has been computed by an implementation of the algorithm described in Section 4 using GNUMP [10] and MPFR [21], within a time equivalent to one day on a single Pentium IV, $4.3 \mathrm{GHz}$.

There are 4113 elements in the table, 1084 if we choose $m_{1}, m_{2} \geq 22$ and 248 if we choose $m_{1}, m_{2} \geq 23$. The maximum distance between two elements of the table is below $2^{-9.977}$, and the worst case is $t_{0}=31$ a93fddd45e3, with $m_{1}, m_{2} \geq 27$. Notice that all these values are very close to what predicts the random model.

We also started the calculation for the double extended precision (64 bits of mantissa) and quadruple precision (113 bits) for $x \in\left[\frac{1}{2}, 1[\right.$. The worst simultaneous cases

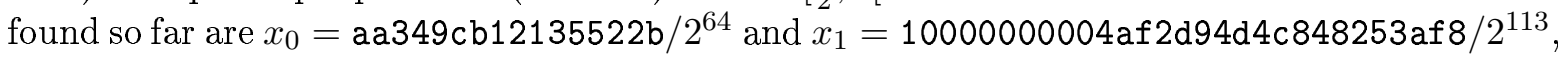
with:

$$
\begin{gathered}
\left|2^{64} \cdot \sin x_{0} \operatorname{cmod} 1\right| \leq 2^{-34} \text { and }\left|2^{64} \cdot \cos x_{0} \operatorname{cmod} 1\right| \leq 2^{-35} \\
\left|2^{114} \cdot \sin x_{1} \operatorname{cmod} 1\right| \leq 2^{-40} \text { and }\left|2^{113} \cdot \cos x_{1} \operatorname{cmod} 1\right| \leq 2^{-40}
\end{gathered}
$$

\section{Acknowledgements}

We thank Vincent Lefèvre for providing the $2^{x}$-table, and Florent de Dinechin and Nicolas Brisebarre for helpful discussions. We also thank the Medicis center for allowing the computation of the $\sin x$ table.

\section{References}

1. The Arenaire Project. Crlibm, 2004. http://lipforge.ens-lyon.fr/projects/crlibm/.

2. N. Brisebarre and J.-M. Muller. Finding the "truncated" polynomial that is closest to a function. INRIA Research Report No 4787, 2003. Updated version available at http://arxiv.org/abs/cs.MS/0307009.

3. D. Coppersmith. Finding a small root of a univariate modular equation. In Proceedings of Eurocrypt'96, volume 1070 of Lecture Notes in Computer Science, pages 155-165. SpringerVerlag, 1996.

4. D. Coppersmith. Finding small solutions to small degree polynomials. In Proceedings of $C A L C^{\prime} 01$, volume 2146 of Lecture Notes in Computer Science, pages 20-31. Springer-Verlag, 2001.

RR $\mathrm{n}^{\circ} 5359$ 


\begin{tabular}{|c|c|c|c|c|c|c|c|c|c|c|c|}
\hline & & & $t_{0}$ & $m_{1}$ & & $t_{0}$ & $m_{1}$ & & 0 & $m_{1}$ & \\
\hline & 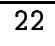 & & & - & & & & 21 & & 21 & \\
\hline & & 26 & & 23 & & & 5 & 21 & & & \\
\hline & & 22 & & 22 & & & 4 & 21 & & & \\
\hline $00 \mathrm{c} 5 \mathrm{c} 52 \mathrm{~b} 1 \mathrm{e}$ & 24 & 22 & $f 00$ & 21 & 6 & ae & 22 & 21 & & , & \\
\hline d30 & 2 & 21 & aa5 & 21 & 5 & & 22 & 22 & & & \\
\hline & & 21 & & 23 & 1 & & 21 & 23 & & & \\
\hline $51 \mathrm{~b}$ & 21 & 21 & 925 & 21 & 25 & Oe & 22 & 21 & 49 & & \\
\hline & 4 & 23 & & 21 & 1 & & 2 & 21 & & & \\
\hline 082241 & 21 & 21 & $6 \mathrm{~b} 2$ & 23 & 23 & 54 & 24 & 22 & $5 b$ & & \\
\hline $64 \mathrm{e}$ & 2 & 21 & & 21 & 1 & & 21 & 21 & & & \\
\hline$c 4 b$ & - & 21 & & 21 & 24 & & 22 & 21 & & & \\
\hline $96 \mathrm{f} 21 \mathrm{a} 2 \mathrm{cba}$ & 23 & 21 & 11 & 21 & 21 & 36 & 23 & 22 & & 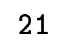 & \\
\hline ff 7 af 85 & 2 & 22 & & 23 & 3 & & 1 & 21 & & & \\
\hline $0 \mathrm{c} 1 \mathrm{acccb} 1200$ & 24 & 22 & Idc & 23 & 24 & $7 a$ & 24 & 25 & & & \\
\hline & 0 & 21 & & 21 & 21 & & 23 & 23 & & & \\
\hline & 21 & 21 & & 21 & 23 & & 22 & 22 & & & \\
\hline $8 \mathrm{f}$ & 21 & 22 & d3 & 22 & 22 & 35 & 22 & 21 & & & 21 \\
\hline & 2 & 23 & & 22 & 1 & & 2 & 22 & & & \\
\hline 655 & 21 & 23 & 78 & 22 & 22 & & 21 & 22 & & & 21 \\
\hline & 21 & 21 & & 22 & 22 & & 22 & 22 & & & 2 \\
\hline c33 & 22 & 21 & & 23 & 21 & & 23 & 24 & & & \\
\hline & 21 & 21 & & 24 & 22 & & 21 & 21 & & & 2 \\
\hline & . & 23 & & 22 & 21 & & 11 & 21 & & & \\
\hline 12 & 24 & 21 & & 21 & 23 & & 23 & 21 & & & 21 \\
\hline & 21 & 22 & & 21 & 22 & & 22 & 22 & & & 2 \\
\hline $\mathrm{a} 8 \mathrm{a}$ & 21 & 21 & & 23 & 26 & & 21 & 21 & & & 23 \\
\hline & 2 & 21 & & 21 & 22 & & 21 & 21 & & & 21 \\
\hline & 21 & 25 & & 22 & 25 & & 21 & 22 & & & 2 \\
\hline & 21 & 27 & & 21 & 21 & & 21 & 24 & & & 21 \\
\hline & 21 & 22 & & 23 & 23 & & 22 & 21 & & & 2 \\
\hline & 23 & 21 & & 21 & 21 & & 23 & 21 & & 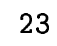 & 22 \\
\hline & & 21 & & 24 & 21 & & 23 & 21 & & & 2 \\
\hline & 22 & 22 & & 23 & 25 & & 24 & 26 & & 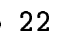 & 2 \\
\hline & & 21 & & 21 & 21 & & 22 & 21 & & & 2 \\
\hline & 21 & 21 & & 22 & 21 & & 22 & 22 & & & 2 \\
\hline & 23 & 23 & & 21 & 22 & & 22 & 21 & & 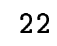 & 22 \\
\hline & 21 & 22 & & 22 & 21 & & 26 & 21 & & & 2 \\
\hline & 2 & 22 & & 21 & 21 & & 22 & 21 & & 22 & 2 \\
\hline & 24 & 24 & & 21 & 21 & & 21 & 22 & & 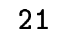 & 22 \\
\hline & 23 & 21 & & 21 & 21 & & 22 & 22 & & 2 & 2. \\
\hline & & 21 & & 22 & 21 & & 21 & 23 & & 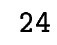 & 2 \\
\hline & 22 & 21 & & 22 & 21 & & 23 & 21 & 11 & 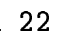 & 21 \\
\hline & 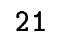 & 21 & & 23 & 22 & & 21 & 21 & & 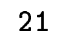 & 25 \\
\hline & 22 & 21 & & 21 & 21 & & 25 & 21 & 111 & 21 & 25 \\
\hline & 22 & 21 & & 23 & 21 & & 22 & 25 & & & 21 \\
\hline & 23 & 23 & & 21 & 21 & & 23 & 21 & & & 21 \\
\hline & 21 & 23 & & 21 & 22 & & 21 & 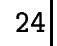 & a8 & 1 & 21 \\
\hline & & & & & & & & & & & \\
\hline
\end{tabular}

INRIA 


\begin{tabular}{|c|c|c|c|c|c|c|c|c|c|c|c|}
\hline$\iota_{0}$ & $m_{1}$ & 2 & $t_{0}$ & $m_{1}$ & & $t_{0}$ & $m_{1}$ & & $t_{0}$ & $m_{1}$ & \\
\hline & 8 & 1 & & 25 & & & 23 & 22 & & 24 & \\
\hline & & 21 & & 23 & & & 22 & & & & \\
\hline & & 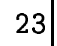 & & 21 & & & 22 & 1 & & & \\
\hline $520 b 39$ & 22 & 21 & $\mathrm{bcc}$ & 23 & 2 & da & 21 & 25 & & & \\
\hline 15 & 1 & 24 & & 21 & 2 & & 21 & 21 & & 1 & \\
\hline $9 d$ & 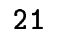 & 21 & & 23 & 1 & & 21 & 21 & & & \\
\hline & 21 & 21 & & 21 & 24 & & 21 & 21 & & & \\
\hline & 1 & 22 & & 21 & 2 & & 1 & 22 & & & \\
\hline 10 & 21 & 21 & 4 & 22 & 22 & & 21 & 21 & 11 & 24 & \\
\hline & & 23 & & 21 & 1 & & 3 & 22 & & & \\
\hline & 2 & 22 & & 22 & 22 & & 22 & 21 & & & \\
\hline & 22 & 25 & & 21 & 21 & & 21 & 21 & ce & 21 & 3. \\
\hline & 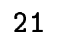 & 22 & & 21 & 2 & & 3 & 21 & & & \\
\hline & 21 & 21 & & 22 & 26 & & 22 & 22 & & 24 & 21 \\
\hline & & 21 & & 21 & 2 & & 21 & 20 & & & ? \\
\hline & 22 & 22 & & 21 & 21 & & 24 & 21 & & - & ? \\
\hline & 21 & 21 & & 22 & 21 & & 23 & 26 & & 24 & 25 \\
\hline & 21 & 22 & & 22 & 21 & & 5 & 21 & & & \\
\hline & 3 & 21 & & 21 & 23 & & 21 & 21 & & 22 & 25 \\
\hline & 2 & 25 & & 22 & 21 & & 21 & 21 & & & 2 \\
\hline & 21 & 25 & & 21 & 25 & & 21 & 26 & & 21 & 23 \\
\hline & 2 & 21 & & 23 & 23 & & 21 & & & 21 & 2. \\
\hline & 21 & 21 & & 21 & 21 & & 1 & 21 & & 22 & 2 \\
\hline & 21 & 21 & & 22 & 21 & & 21 & 27 & & 3 & 21 \\
\hline & 21 & 22 & & 21 & 21 & & 23 & 21 & & 2 & 2 \\
\hline & 23 & 22 & & 21 & 21 & & 23 & 21 & & 6 & 24 \\
\hline & $\Omega$ & 24 & & 21 & 21 & & 22 & 21 & & 25 & 2. \\
\hline & 2 & 21 & & 22 & 22 & & 21 & 21 & & 1 & 23 \\
\hline & O & 22 & & 22 & 21 & & 24 & 21 & & 22 & 22 \\
\hline & 2 & 21 & & 21 & 21 & & 23 & 23 & & 22 & 21 \\
\hline & & 21 & & 21 & 22 & & 24 & 22 & & 2 & 22 \\
\hline & 21 & 21 & & 21 & 21 & & 22 & 22 & & 22 & 22 \\
\hline & 2 & 21 & & 22 & 22 & & 21 & 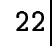 & & 21 & 21 \\
\hline & 22 & 21 & & 21 & 23 & & 24 & 21 & & 21 & 22 \\
\hline & 25 & 22 & & 24 & 24 & & 24 & 21 & & 23 & 21 \\
\hline & 22 & 21 & & 21 & 22 & & 23 & 21 & & 1 & 23 \\
\hline 53 & 21 & 32 & 52 & 21 & 22 & $c 6$ & 21 & 22 & $=47$ & 21 & 22 \\
\hline
\end{tabular}

Fig. 2. The smallest 340 entries of the table for $\sin x$ for double precision and $x \in\left[\frac{1}{2}, 1[\right.$ : for any entry, $t_{0}, m_{1}, m_{2}$ satisfy equation (3). 
5. D. Defour. Cache-optimized methods for the evaluation of elementary functions. LIP Research Report RR2002-38, 2002. Available at ftp://ftp.ens-lyon.fr/pub/LIP/Rapports/RR/RR2002/RR2002-38.ps.Z.

6. D. Defour, F. de Dinechin, and J.-M. Muller. Correctly rounded exponential function in double precision arithmetic. In Proceedings of SPIE 46th Annual Meeting, International Symposium on Optical Science and Technology, 2001.

7. D. Defour, G. Hanrot, V. Lefèvre, J.-M. Muller, N. Revol, and P. Zimmermann. Proposal for a standardization of mathematical function implementation in floating-point arithmetic. Numerical Algorithms. To appear.

8. S. Gal. Computing elementary functions: a new approach for achieving high accuracy and good performance. In Proceedings of Accurate Scientific Computations, volume 235 of Lecture Notes in Computer Science, pages 1-16. Springer-Verlag, 1986.

9. S. Gal and B. Bachelis. An Accurate Elementary Mathematical Library for the IEEE Floating Point Standard. ACM Transactions on Mathematical Software, 17(1):16-45, 1991.

10. T. Granlund. GNU MP: The GNU Multiple Precision Arithmetic Library, 4.1 .4 edition, 2004. Available at http://ww.swox.se/gmp/.

11. P. M. Gruber and C. G. Lekkerkerker. Geometry of Numbers, second edition. Amsterdam, North-Holland, 1987.

12. IEEE standard for binary floating-point arithmetic. Technical Report ANSI-IEEE Standard 754-1985, New York, 1985.

13. V. Lefèvre. Moyens arithmétiques pour un calcul fiable. Thèse de doctorat, École Normale Supérieure de Lyon, 2000.

14. V. Lefèvre and J.-M. Muller. Worst cases for correct rounding of the elementary functions in double precision. In Proceedings of the 15th IEEE Symposium on Computer Arithmetic, pages 111-118. IEEE Computer Society, 2001.

15. A. K. Lenstra, H. W. Lenstra, and L. Lovász. Factoring Polynomials with Rational Coefficients. Mathematische Annalen, 261:515-534, 1982.

16. L. Lovász. An Algorithmic Theory of Numbers, Graphs and Convexity. SIAM lecture series, $50,1986$.

17. J.-M. Muller. Elementary Functions, Algorithms and Implementation. Birkhauser Boston, 1997.

18. J.-M. Muller. Proposals for a specification of the elementary functions. In Abstracts of SCAN'2002, pages 54-55. Laboratory LIP6, Paris, France, 2002.

19. P. Nguyen and D. Stehlé. Low-dimensional lattice basis reduction revisited (extended abstract). In Proceedings of ANTS VI, volume 3076 of Lecture Notes in Computer Science, pages 338-357. Springer-Verlag, 2004.

20. M. Payne and R. Hanek. Radian reduction for trigonometric functions. SIGNUM Newsletter, 18:19-24, 1983.

21. The Spaces Project. The MPFR library, version 2.0.3, 2004. http://www.mpfr.org/.

22. D. Stehlé, V. Lefèvre, and P. Zimmermann. Worst cases and lattice reduction. In Proceedings of the 16th IEEE Symposium on Computer Arithmetic, pages 142-147. IEEE Computer Society, 2003.

23. D. Stehlé, V. Lefèvre, and P. Zimmermann. Searching Worst Cases of a One-Variable Function. To appear in IEEE Transactions on Computers, 2005.

24. A. Ziv. Fast Evaluation of Elementary Mathematical Functions with Correctly Rounded last Bit. ACM Transactions on Mathematical Software, 17(3):410-423, 1991.

25. A. Ziv. MathLib, 2004. http://www-124.ibm.com/developerworks/oss/mathlib/. 


\section{Appendix:}

We study here the expected maximum distance between two bad cases of a function $f$ under the random model.

Lemma 3. Let $f:\left[\frac{1}{2}, 1\left[\rightarrow\left[\frac{1}{2}, 1[\right.\right.\right.$ satisfying the random model assumption. Let $n$ be the precision and $2 \leq p \leq n$. Let $M$ be the maximum distance between two consecutive $p$-bad cases for $f$. We have:

$$
E[M] \leq\left(1+n-\frac{p}{2}\right) \cdot 2^{p-n}
$$

Proof: The probability of having a run of at least $k$ consecutive machine numbers that are not $p$-bad cases for $f$ is bounded by:

$$
2^{n-1} \cdot\left(1-2^{1-p}\right)^{k}
$$

because there are less than $2^{n-1}$ starting points for the run. We now fix $k=$ $\left(n-1-\frac{p}{2}\right) \ln 2 \cdot 2^{p}$. Using the fact that $\log (1-x) \leq-x-x^{2}$ for any $x \in\left[0, \frac{1}{2}\right]$, we can bound the probability above by:

$2^{n-1+2^{p}\left(n-1-\frac{p}{2}\right) \log \left(1-2^{1-p}\right)} \leq 2^{n-1-2^{p}\left(n-1-\frac{p}{2}\right)\left(2^{1-p}+2^{2-2 p}\right)} \leq 2^{p-n+1} \cdot 2^{2^{2-p}} \leq 2 \cdot 2^{p-n+1}$,

from which we obtain that:

$$
\begin{aligned}
E[M] & \leq k \cdot 2^{-n}+\frac{1}{2} \cdot 2 \cdot 2^{p-n+1} \\
& \leq 2^{p-n} \cdot\left(n+1-\frac{p}{2}\right)
\end{aligned}
$$

which ends the proof.

RR $n^{\circ} 5359$ 


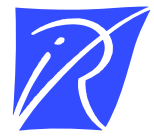

Unité de recherche INRIA Lorraine

LORIA, Technopôle de Nancy-Brabois - Campus scientifique 615, rue du Jardin Botanique - BP 101 - 54602 Villers-lès-Nancy Cedex (France)

Unité de recherche INRIA Futurs : Parc Club Orsay Université - ZAC des Vignes

4, rue Jacques Monod - 91893 ORSAY Cedex (France)

Unité de recherche INRIA Rennes : IRISA, Campus universitaire de Beaulieu - 35042 Rennes Cedex (France)

Unité de recherche INRIA Rhône-Alpes : 655, avenue de l'Europe - 38334 Montbonnot Saint-Ismier (France) Unité de recherche INRIA Rocquencourt : Domaine de Voluceau - Rocquencourt - BP 105 - 78153 Le Chesnay Cedex (France)

Unité de recherche INRIA Sophia Antipolis : 2004, route des Lucioles - BP 93 - 06902 Sophia Antipolis Cedex (France)

INRIA - Domaine de Voluceau - Rocquencourt, BP 105 - 78153 Le Chesnay Cedex (France)

http://www.inria.fr

ISSN 0249-6399 